ARTICLE

Received 18 Dec 2013 | Accepted 17 Jun 2014 | Published 18 Jul 2014 DOl: 10.1038/ncomms5422

\title{
Tabletop imaging of structural evolutions in chemical reactions demonstrated for the acetylene cation
}

Heide Ibrahim', Benji Wales², Samuel Beaulieu', Bruno E. Schmidt', Nicolas Thiré1, Emmanuel P. Fowe ${ }^{3}$, Éric Bisson ${ }^{1}$, Christoph T. Hebeisen ${ }^{4,5}$, Vincent Wanie', Mathieu Giguére ${ }^{1}$, Jean-Claude Kieffer ${ }^{1}$, Michael Spanner ${ }^{4}$, André D. Bandrauk ${ }^{3}$, Joseph Sanderson², Michael S. Schuurman ${ }^{4}$ \& François Légaré ${ }^{1}$

The introduction of femto-chemistry has made it a primary goal to follow the nuclear and electronic evolution of a molecule in time and space as it undergoes a chemical reaction. Using Coulomb Explosion Imaging, we have shot the first high-resolution molecular movie of a to and fro isomerization process in the acetylene cation. So far, this kind of phenomenon could only be observed using vacuum ultraviolet light from a free-electron laser. Here we show that $266 \mathrm{~nm}$ ultrashort laser pulses are capable of initiating rich dynamics through multiphoton ionization. With our generally applicable tabletop approach that can be used for other small organic molecules, we have investigated two basic chemical reactions simultaneously: proton migration and $\mathrm{C}=\mathrm{C}$ bond breaking, triggered by multiphoton ionization. The experimental results are in excellent agreement with the timescales and relaxation pathways predicted by new and quantitative $a b$ initio trajectory simulations.

\footnotetext{
${ }^{1}$ Institut National de la Recherche Scientifique, Centre Énergie Matériaux et Télécommunications, 1650 Boulevard Lionel-Boulet, Varennes, Quebec, Canada J3X 1S2. ${ }^{2}$ Department of Physics and Astronomy, University of Waterloo, 200 University Avenue West, Waterloo, Ontario, Canada N2L 3G1. ${ }^{3}$ Laboratoire de Chimie Théorique, Faculté des Sciences, 2500, Boulevard de I'Université, Université de Sherbrooke, Quebec, Canada J1K 2R1. ${ }^{4}$ National Research Council of Canada, 100 Sussex Dr, Ottawa, Ontario, Canada K1A 0R6. ${ }^{5}$ Department of Physics, University of Ottawa, 150 Louis Pasteur, Ottawa Ontario, Canada K1N 6N5. Correspondence and requests for materials should be addressed to H.I. (email: ibrahim@emt.inrs.ca) or to F.L. (email: legare@emt.inrs.ca).
} 
S ince the introduction of femto-chemistry ${ }^{1-5}$, electron or $\mathrm{X}$-ray diffraction have been the most commonly used techniques to track nuclear rearrangement in molecules ${ }^{6-9}$. Unfortunately, these techniques are largely insensitive to the more subtle and irregular structural changes that can occur within a single small molecule undergoing a chemical reaction. Pump-probe Coulomb Explosion Imaging (CEI) allows observation of these changes on a femtosecond ( $\mathrm{fs}$ ) timescale with atomic resolution ${ }^{10-13}$. So far, however, important phenomena such as proton migration in the acetylene cation have only been observed using vacuum ultraviolet light (VUV) from a free-electron laser (FEL) ${ }^{14}$.

Since laser-driven tunnel ionization preferentially ionizes a neutral molecule to the electronic ground state of the cation, any subsequent nuclear dynamics will generally occur on that state. The ability to readily initiate dynamics on an excited electronic state of the cation would enable a variety of new and complex experiments. Using the intuitive Koopman's approximation picture of ionization ${ }^{15}$, the ground electronic state of a cation is generated by removal of an electron from the highest molecular orbital (HOMO) of the neutral, whereas excited states are generated by ionizing electrons from the lower-lying HOMO-n orbitals. It had, for example, been assumed that only ionization of the HOMO contributes to high harmonic generation (HHG) because of the low frequency fields used. However, recent results, obtained at $800 \mathrm{~nm}$, demonstrate that ionization of HOMO-n orbitals may also contribute to an HHG spectrum ${ }^{16-18}$. Given the coherent nature of HHG process, even a small excited state contribution can have a significant impact on the observed signal due to interference effects. However, in CEI, these contributions add linearly, and thus a small contribution from ionization of the HOMO-n would be difficult to observe.

To efficiently launch dynamics in excited states of molecules with a large energy gap between ground and excited states, it is common to use high photon energy sources such as FELs

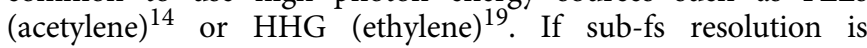
required, one can use an XUV pump/XUV probe arrangement based on HHG for this kind of experiments ${ }^{20,21}$, however, at the price of a highly demanding experimental setup.

Even though acetylene $\left(\mathrm{C}_{2} \mathrm{H}_{2}\right)$ has been serving as a model system for decades, proton migration dynamics in its cation is not yet fully elucidated, and has recently attracted attention of both experimental and theory groups ${ }^{12-14,22-24}$. Given its small size, it is an ideal candidate for the application of quantitative electronic structure theory, which can be brought to bear on its ground and excited states.

Actually, proton migration pumped by $800 \mathrm{~nm}$ pulses has been observed solely in the dication of deuterated acetylene by Hishikawa and co-workers ${ }^{12,13}$. Very recently, optimal control experiments confirmed that excitation with $800 \mathrm{~nm}$ pulses leads to isomerization in the dication ${ }^{25}$. These $800 \mathrm{~nm}$ pump schemes, however, are not capable of launching proton migration in other charge states such as in the cation ${ }^{26}$.

This is because it is quite challenging to overcome the potential energy barrier of $2 \mathrm{eV}$ (ref. 27) that separates the acetylene cation $[\mathrm{HC}=\mathrm{CH}]^{+}$in its ground state $X^{2} \Pi_{\mathrm{u}}$ from vinylidene. Therefore, to obtain sufficient energy to overcome this isomerization barrier ${ }^{22}$, it is necessary to populate the A-state $\left(\mathrm{A}^{2} \Sigma_{\mathrm{g}}^{+}\right)$of $[\mathrm{HC}=\mathrm{CH}]^{+}(5.3 \mathrm{eV}$ above the ground state $)$, which requires ionization from the HOMO-1. This cannot be achieved in the adiabatic regime with $800 \mathrm{~nm}$ pulses ${ }^{26}$. To remain in the adiabatic regime for long conjugated molecules that display small energy gaps, Lezius et al. ${ }^{28}$ have suggested increasing the laser wavelength. Inverting this idea, it appears sensible to reach the non-adiabatic regime for small molecules by using UV wavelengths with larger photon energies.
We present a generalized approach using $266 \mathrm{~nm}$ pump-pulses that unites the convenience and flexibility of a tabletop setup with excitation ranges of FELs, and unveils the isomerization process in unprecedented detail allowing us to record a high-resolution molecular movie of proton migration from the linear acetylene cation $\left([\mathrm{HC}=\mathrm{CH}]^{+}\right)$to the vinylidene cation $\left(\left[\mathrm{C}=\mathrm{CH}_{2}\right]^{+}\right)$. This movie shows that multiple oscillations can occur between the two isomers acetylene and vinylidene, revealing that results obtained in a tabletop multiphoton approach can go well beyond the FEL results ${ }^{14}$. Thus, multiphoton ionization with ultrashort UV laser pulses provides a powerful alternative to demanding VUV sources. This paper is structured as follows: first, in a singlepulse experiment, we verify that excitation with $266 \mathrm{~nm}$ pulses leads to the same spectral signatures as those obtained with an $\mathrm{FEL}^{14}$, confirming that we populate the first excited electronic state of the acetylene cation $\left(\mathrm{A}^{2} \Sigma_{\mathrm{g}}^{+}\right)$that initiates proton migration. Second, we present time-resolved pump-probe

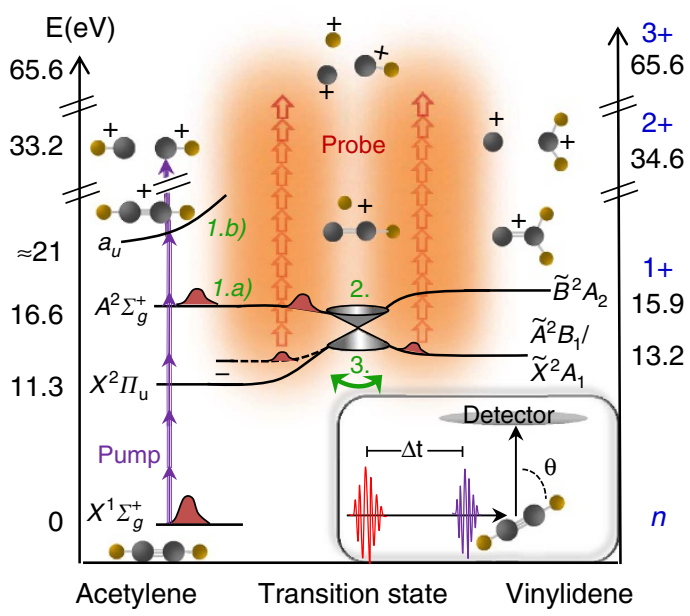

Figure 1 | Experimental scheme: (1.a) a 266-nm pump-pulse populates the first excited state $A^{2} \Sigma_{g}^{+}$of $[\mathrm{HC}=\mathrm{CH}]^{+}$in a four-photon ionization process. Proton migration pathway: (2) within $40 \mathrm{fs}$, the wavepacket enters a region of strong non-adiabatic coupling with the ground $X^{2} \Pi_{u}$ state of $[\mathrm{HC}=\mathrm{CH}]^{+}(1+)$. Passage through this region involves relaxation to the $X$-state via a conical intersection. (3) Following this transition, the molecule, now in a highly vibrationally excited state, may undergo isomerization to the vinylidene cation $\left[\mathrm{C}=\mathrm{CH}_{2}\right]^{+}$. A to and fro isomerization between acetylene and vinylidene takes places, indicated by the green double arrow. Dissociation pathway: (1.b) while the 32-fs pump-pulse is present, a fifth photon can be absorbed on the A-state thus exciting population to higher lying states (presumably of $a_{u}$ symmetry; Supplementary Fig. 1) that have sufficient energy to dissociate along the $\mathrm{C}=\mathrm{C}$ bond. Pictograms show the molecular structure in each configuration, starting from neutral acetylene $(n)$ in the lower left corner. After excitation, $[\mathrm{HC}=\mathrm{CH}]^{+}$remains in linear configuration $\left(D_{\infty h}\right.$ ), reaches the trans-geometry (Fig. 5), followed by the transition state and changes to a ' $Y$-shape' once a proton migrates from one $\mathrm{C}$-atom to the other in the vinylidene structure $\left(\mathrm{C}_{2 v}\right)$. To probe the nuclear structure, either further $266 \mathrm{~nm}$ photons are absorbed to reach the dication of acetylene and vinylidene (single-pulse experiment) or a second timedelayed $800 \mathrm{~nm}$ pulse leads to Coulomb explosion of the charged molecule (pump-probe experiment). With the latter, we reach the doubly charged $(2+)$ as well as the triply charged ionic states $(3+)$, and thus correlate either two fragments $\left(\mathrm{CH}^{+}+\mathrm{CH}^{+}\right.$or $\left.\mathrm{C}^{+}+\mathrm{CH}_{2}^{+}\right)$or three fragments $\mathrm{H}^{+}+\mathrm{C}^{+}+\mathrm{CH}^{+}$with each other. In the inset, a sketch of the experimental setup with orientations of molecules, laser pulses and time of flight (TOF) direction towards the detector is given, with $\theta$ being the angle between molecular axis and laser polarization. Molecules being oriented parallel to the laser field correspond to $\theta=0^{\circ}$. Energy levels are taken from ref. 27 and our calculations. 
experiments of the two-body breakup. Based on the single-pulse experiments, the presence of $\mathrm{CH}_{2}^{+}$in the dynamics of the vinylidene channel in the cation $\left(\mathrm{C}^{+}+\mathrm{CH}_{2}^{+}\right.$correlation) is a clear indicator of proton migration since one proton has been migrating to the other side of the molecule. In addition to proton migration resulting in the formation of bound vinylidene molecules in the cation, new channels that lead to dissociation of the cation are observed in both, the vinylidene and the acetylene $\left(\mathrm{CH}^{+}+\mathrm{CH}^{+}\right)$correlations. And finally, we investigate the three-fragment correlations from the same experimental data set $\left(\mathrm{C}^{+}+\mathrm{CH}^{+}+\mathrm{H}^{+}\right)$and construct a molecular movie of proton migration that exhibits excellent agreement with new numerical simulations of non-adiabatic wavepacket propagation on the ground and excited electronic states of the cation. These $a b$ initio trajectory simulations use definitive levels of electronic structure theory in the description of the relevant potential energy surfaces.

\section{Results}

Single-pulse experiment. Signatures of proton migration in $\mathrm{C}_{2} \mathrm{H}_{2}^{+}$have recently been demonstrated at the FEL in Hamburg ${ }^{14}$ with XUV light $(38 \mathrm{eV})$. Indications of time-dependent acetylenevinylidene isomerization, photoinitiated via ionization to the A-state of the acetylene cation appear in the kinetic energy release (KER) of correlated $\mathrm{C}^{+}$and $\mathrm{CH}_{2}^{+}$fragments at energies above $5.8 \mathrm{eV}$ (ref. 14). As an initial experiment, we set out to find this signature by using single laser pulses of $266 \mathrm{~nm}$ central wavelength and varying pulse duration and peak power. The experimental scheme in terms of relevant electronic and ionic states and excitation pathways is described in Fig. 1. The A-state of the acetylene cation is reached by absorption of four $266 \mathrm{~nm}$ photons (corresponding to $4 \times 4.66 \mathrm{eV}=18.64 \mathrm{eV}$ ). Once on the A-state, proton migration occurs, which is then followed by ionization to a dissociative state of the dication within the same laser pulse. Results of a single $266-\mathrm{nm}$ pulse experiment are shown in Fig. 2a), containing the KER distributions of the twofragment correlation channel of vinylidene for $32 \mathrm{fs}$ (green and red), $110 \mathrm{fs}$ (black) and $250 \mathrm{fs}$ (blue) pulse duration and various pulse powers. Already a short pulse of moderate pulse power (green solid line) leads to a KER distribution broadened on the high-energy side. With increasing pulse duration, a strongly pronounced high-energy shoulder appears. The shoulder obtained with $250 \mathrm{fs}$ pulses (blue solid line) agrees very well with data obtained at the FEL using $38 \mathrm{eV}$ (open circles) ${ }^{14}$. This shoulder was assigned to proton migration initiated by ionizing acetylene to the A-state of its cation ${ }^{14,22,24,29}$. The proton migration that we observe is essentially pulse duration dependent and only secondarily intensity dependent. The intensities of the short $32 \mathrm{fs}$ pulses $\left(3 \times 10^{14}\right.$ and $6 \times 10^{14} \mathrm{~W} \mathrm{~cm}^{-2}$ ), which are higher by a factor of five than those for the long pulses, indicate that increasing the intensity alone will not lead to a strongly pronounced high-energy shoulder. Fragmentation occurs in the doubly charged state that is accessed here in a sequential process within a single pulse; this means the complete sequence, of first pump followed by proton migration and finally probe, is taking place underneath the envelope of the 266-nm pulse and we observe the accumulated signal of molecules that have undergone the full sequence. This effect is therefore most pronounced for the longest pulses ( $250 \mathrm{fs}$ ) that are also of the lowest intensity.

In addition, we used $800 \mathrm{~nm}, 200 \mathrm{fs}$ pulses (grey shaded). The energetically narrow KER distribution from $800 \mathrm{~nm}$ excitation confirms the observation of Alnaser et al. ${ }^{26}$ In this case, we do not obtain high-energy fragments as a signature of isomerization in the acetylene cation, and similar results were obtained at $400 \mathrm{~nm}$ (not shown). This was a primary motivation for our study at $266 \mathrm{~nm}$ in order to confirm that UV excitation is essential to populate the A-state.

Initial state assignment (I). Assignment of the electronic states involved in the isomerization can be more demanding in a multiphoton scheme compared with a single photon scheme pumped by one VUV photon. In both cases, however, multiple states can be populated, for example, the ground and the first excited state of the cation. In our experiment, while the cationic A-state is excited by four photons, the ground state of the cation $X^{2} \Pi_{u}$ is populated in a three-photon process. However, the documented energy barrier separating the acetylene and

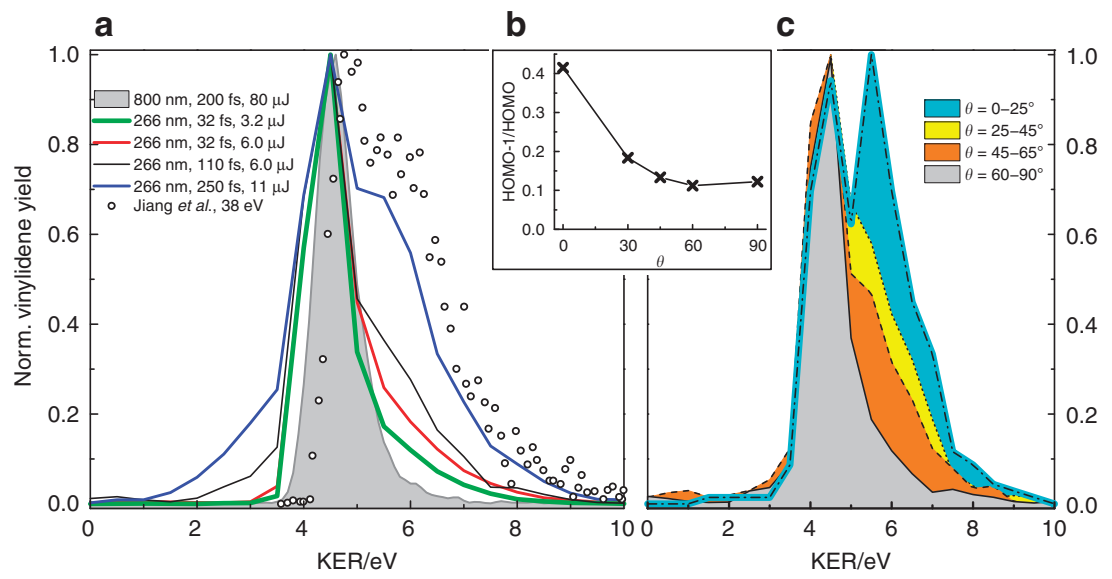

Figure 2 | Single-pulse experiment. (a) Wavelength, pulse duration and energy dependence (with estimated intensities) on the vinylidene yield versus total KER $\left(\mathrm{C}^{+}+\mathrm{CH}_{2}^{+}\right)$. Filled grey curve: $800 \mathrm{~nm}$ excitation, $200 \mathrm{fs}$ pulse duration, $80 \mu \mathrm{J}, 4 \times 10^{14} \mathrm{~W} \mathrm{~cm}^{-2}$; green curve: $266 \mathrm{~nm}$ excitation, $32 \mathrm{fs}$ pulse duration, $3.2 \mu \mathrm{J}, 3 \times 10^{14} \mathrm{~W} \mathrm{~cm}^{-2}$; red curve: $266 \mathrm{~nm}$ excitation, $32 \mathrm{fs}$ pulse duration, $6.0 \mu \mathrm{J}, 6 \times 10^{14} \mathrm{~W} \mathrm{~cm}^{-2}$; black curve: $266 \mathrm{~nm}$ excitation, $110 \mathrm{fs}$ pulse duration, $6.0 \mu \mathrm{J}, 1 \times 10^{14} \mathrm{~W} \mathrm{~cm}^{-2}$; blue curve: $266 \mathrm{~nm}$ excitation, $250 \mathrm{fs}$ pulse duration, $11 \mu \mathrm{J}, 1 \times 10^{14} \mathrm{~W} \mathrm{~cm}^{-2}$; open circles: excitation with $38 \mathrm{eV}$ from an $\mathrm{FEL}^{14}$. (b) TDDFT calculations showing the ratio of ionization rate of $\mathrm{HOMO}-1$ to $\mathrm{HOMO}$ versus angle $\theta$. Pulse parameters were: wavelength: $266 \mathrm{~nm}$, pulse duration: $24 \mathrm{fs}$ ( 27 cycles), intensity: $3 \times 10^{14} \mathrm{Wcm}^{-2}$. (c) Cuts of the angular distribution of the $266 \mathrm{~nm}, 110 \mathrm{fs}$ spectrum shown as black solid line in a. Cyan corresponds to angles $\theta$ between $0-25^{\circ}$ (note that the high-energy peak exceeds the main peak here); yellow corresponds to $25-45^{\circ}$, orange to $45-$ $60^{\circ}$ and grey to $60-90^{\circ}$. All curves are normalized to the peak maximum. 
vinylidene isomers will prohibit proton migration from low-lying vibrational levels on the electronic ground state $^{27}$, so its contribution will only show up around the main peak of KER in Fig. 2a). To confirm that the experimentally observed highenergy shoulder arises from ionization to the A-state ${ }^{14}$, we investigate the angular distribution of the molecular fragments from the KER spectrum obtained with the $110 \mathrm{fs}, 266 \mathrm{~nm}$ pulse shown as a black solid line in Fig. 2a). Initially, all population is in the $\mathrm{X}^{1} \Sigma_{g}^{+}$electronic state of the neutral acetylene. Following Koopman's approximation ${ }^{15}$, ionization to the $\mathrm{A}^{2} \Sigma_{g}^{+}$state of the acetylene cation requires the removal of one electron from the HOMO- 1 , which is a $\sigma_{\mathrm{g}}$ orbital. In general, the probability for ionization from this orbital is largest for those molecules aligned parallel to the polarization of the laser field ${ }^{17}$ (this corresponds to the angle $\theta=0^{\circ}$, consistent with the definition given in the inset of Fig. 1). Our experimental situation of strong field multiphoton ionization represents an uncommon case located between weak field multiphoton ionization and tunnel ionization. State of the art literature ${ }^{30}$ proposes time-dependent density functional theory (TDDFT) to represent exactly this situation. Our TDDFT calculations of the ionization rates of HOMO-1 and HOMO confirm the anticipated angular dependence. As expected, their ratio in Fig. 2b) shows a well-pronounced maximum for angle $\theta=0^{\circ}$. Note that this is also exactly where the maximum in the high-energy shoulder of the angularresolved KER distribution is observed (see cyan shaded curve of Fig. 2c). The high-energy maximum of this particular angular slice is significantly more pronounced than for all other angular distributions and even exceeds the main peak. Thus, we can confidently assign this region to ionization to the $\mathrm{A}^{2} \Sigma_{g}^{+}$state. Although higher electronic states with $\Sigma$ symmetry exist in the cation, the probability of them being populated under the current experimental condition is significantly lower than for the A-state (Supplementary Discussion).

Pump-probe study of the two-body breakup process. Next, we used pump-probe CEI to image the dynamics resulting from multiphoton ionization with $266 \mathrm{~nm}$ pulses. We investigate two chemical reactions: (i) proton migration-one of the fundamental processes in chemistry and biology; and (ii) the first observation of $\mathrm{C}=\mathrm{C}$ bond breaking on highly excited states of the acetylene and vinylidene cation.

We focus first on the isomerization process: as shown in the experimental scheme of Fig. 1, a 266-nm pump-pulse ionizes the system to $[\mathrm{HC}=\mathrm{CH}]^{+}$in a four-photon process, thereby initiating proton migration dynamics. A time-delayed, $800-\mathrm{nm}$ probe-pulse tunnel ionizes the molecule to doubly and triply charged states of acetylene or vinylidene. Its electric field stripes off electrons, leaving behind positively charged fragments that undergo Coulomb explosion. As in the single-pulse experiments, proton migration dynamics shows up in pump-probe experiments in the high-energy region obtained by correlating two fragments. This dynamics corresponds to the region $\mathrm{V} 3$ of the vinylidene channel $\left(\mathrm{C}^{+}+\mathrm{CH}_{2}^{+}\right)$in Fig. 3a. We observe an increase of the yield in this high-energy shoulder in the temporal window of $60-100 \mathrm{fs}$ followed by a decrease. (We will leave the details about the proton migration dynamics and vinylidene formation until we have direct evidence from three-fragment correlations in the next section.) Note that this high-energy shoulder is less pronounced in the pump-probe experiment of Fig. 3 compared with the single-pulse experiment in Fig. 2a), in which the complete two-step ionization process occurs many times beneath a single-pulse envelope. Therefore, for long singlepulse durations (110 and $250 \mathrm{fs}$ ), the excited state dynamics can be initiated on a larger number of molecules and this gives rise to
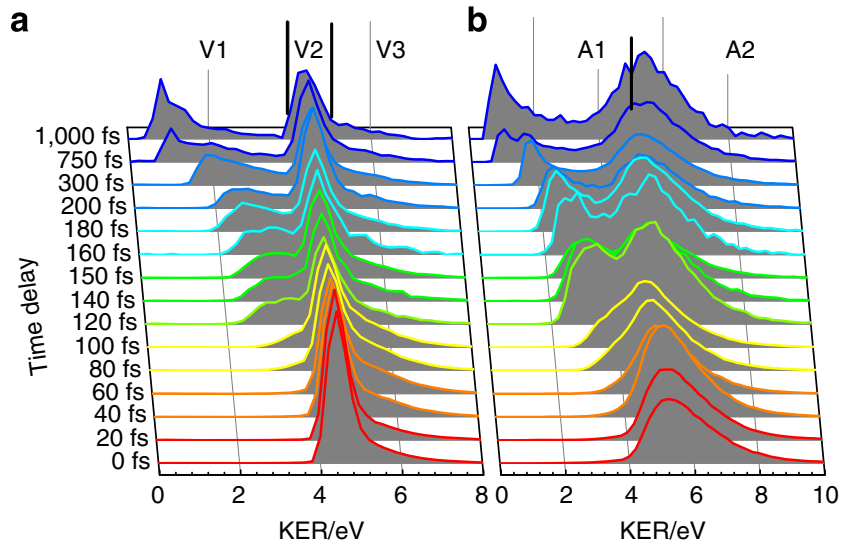

Figure 3 | Two-fragment correlation-total KER. Vinylidene channel $\mathrm{C}^{+}+\mathrm{CH}_{2}^{+}$is shown in $\mathbf{a}$ and acetylene channel $\mathrm{CH}^{+}+\mathrm{CH}^{+}$in $\mathbf{b}$ with increasing time delay from $0 \mathrm{fs}$ (red) to 1,000 fs (blue). The vinylidene spectrum contains three different regions: $\mathrm{V} 1$ : the low-energy range below $4 \mathrm{eV}$; V2: the intermediate energy range between 4 and $5 \mathrm{eV}$; and V3: the high-energy range, $>5 \mathrm{eV}$. The acetylene channel is divided into two regions: the low-energy region $A 1:<4 \mathrm{eV}$, and the high-energy region $\mathrm{A} 2$ : $>4 \mathrm{eV}$. Spectra are normalized by the number of correlated counts, to account for varying acquisition times at different time steps, as well as for minor fluctuations in pulse power or gas pressure. This normalization allows comparison of the absolute count number in each channel at each time delay; energy binning and total number of counts per time step are given in Supplementary Table 1.

an accumulated vinylidene formation compared with the 32 -fs in case of the pump-probe experiment.

The mid-energy region V2 in Fig. 3 a has been previously ${ }^{14}$ assigned to a sequential double ionization. In addition, we observe a new peak emerging in the low-energy region V1. This peak is first visible around $80 \mathrm{fs}$, and occurs as well in the lowenergy region $\mathrm{A} 1$ of the acetylene channel $\left(\mathrm{CH}^{+}+\mathrm{CH}^{+}\right.$; Fig. $3 \mathrm{~b}$ ) and has, to our knowledge, never been observed before for $\mathrm{C}_{2} \mathrm{H}_{2}^{+}$. Since it occurs for both vinylidene and acetylene, we attribute it to a stretching of the $\mathrm{C}=\mathrm{C}$ bond. In both channels, it migrates to smaller energies (indicating an increase in the $\mathrm{C}-\mathrm{C}$ internuclear distance) with increasing time delay. The peak strength continuously increases with increasing time delay, which is consistent with a lowering of the ionization potential as the $\mathrm{C}=\mathrm{C}$ bond length increases. We separate the KER structure of the acetylene channel into only two areas, A1 and A2, since no proton migration is observed in this channel. A2 contains contributions from a direct ionization to the dication as well as a subsequent ionization from the cation to the dication, and is not further investigated in this paper.

Using Coulomb potentials for analysis, the peak at $1 \mathrm{eV}$ corresponds to an elongation of the $\mathrm{C}=\mathrm{C}$ bond to $>4 r_{\mathrm{eq}}$, where $r_{\mathrm{eq}}$ is the equilibrium distance, implying that the molecule has largely dissociated. Similar dissociation effects have been observed in $\mathrm{C}_{2} \mathrm{H}_{2}^{2+}$ (ref. 31) and 1,3-butadiene ${ }^{32}$. While none of the lowlying electronic states of $\mathrm{C}_{2} \mathrm{H}_{2}^{+}$is dissociative along the $\mathrm{C}-\mathrm{C}$ stretch coordinate, if a fifth photon is absorbed during the pumppulse, the molecule will have energy in excess of the lowest $\mathrm{C}-\mathrm{C}$ dissociation asymptotes (Fig. 1 and Supplementary Fig. 1). Since the dissociation dynamics occur in both the acetylene and vinylidene channels, they are likely to be triggered by the same event, namely absorption of a photon to higher lying excited states of the cation. From there, it can proceed to dissociative states of the vinylidene cation. This dissociation pathway has not been observed at a FEL ${ }^{14}$, given that for one-photon absorption, 


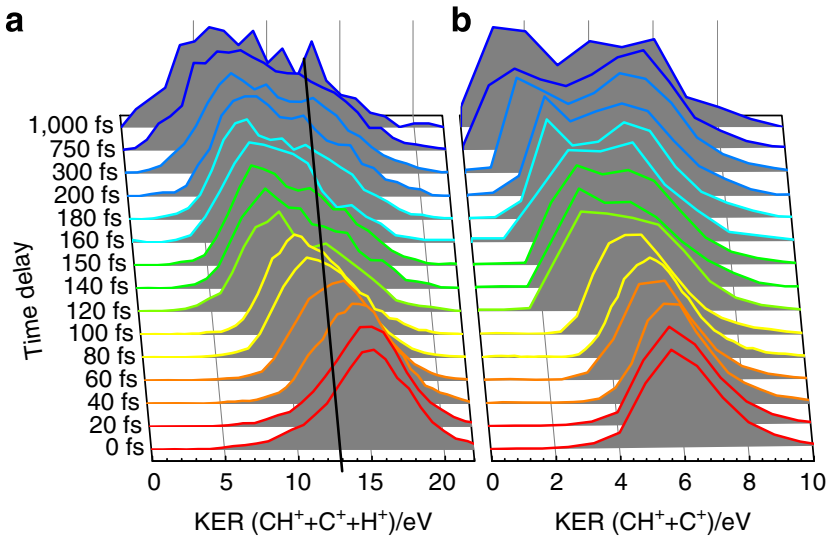

Figure 4 | Three-fragment correlation data-KER. Peak values are normalized to the maximum. (a) Total KER $\mathrm{CH}^{+}+\mathrm{C}^{+}+\mathrm{H}^{+}$; (b) KER from $\mathrm{CH}^{+}+\mathrm{C}^{+}$fragments. The black line in a indicates the energy cutoff for the Newton plots of Fig. 5. Binning was adjusted according to the number of correlated counts available for each time delay. Note that the data for a time delay of 1 ps contain significantly less statistics than other time delays, displayed sub-maxima are related to this.

once ionized, no further coupling between electronic states occurs. We predict that in a multiphoton experiment with shorter pump-pulses, this sequential coupling after ionization is also likely to be suppressed.

Pump-probe study of the three-body breakup process. This new dissociation channel also shows up in the three-body breakup $\mathrm{C}_{2} \mathrm{H}_{2}^{3+} \rightarrow \mathrm{H}^{+}+\mathrm{C}^{+}+\mathrm{CH}^{+}$, in which two electrons are removed by the probe-pulse. Before we address the rich information present in this channel though, we first need to prove that it is indeed what we claim, and that the probe-pulse is only showing us dynamics of the cation. As a first step in doing so, we compare the KER features in the two-body channel (Fig. 3), which we have established to show us time-dependent processes that must originate in the cation, with those of the three-body channel in Fig. 4. Figure 4a contains the total KER during the Coulomb explosion. The peak maximum migrates from $\sim 15 \mathrm{eV}$ at time zero to $7 \mathrm{eV}$ at a time delay of a picosecond, indicating the presence of the dissociative channel described in the previous section. To uncover the close analogy between two- and threebody breakup, we take advantage of the fact that in the threebody channel an $\mathrm{H}^{+}$fragment is created, which, because of conservation of momentum, is responsible for carrying away most of the increased and broadband energy release. If the $3+$ channel is observing the same dynamics, we expect strong similarities in the energy sum of correlated $\mathrm{C}^{+}+\mathrm{CH}^{+}$fragments of the three-body channel, with the total energy released in the twobody channel. To extract hidden sub-structures of Fig. 4a, we look at the $3+$ KER reduced by the proton energy in Fig. 4b, leading to a double-peak structure with one peak $\sim 6 \mathrm{eV}$, and another one emerging for longer times and moving to $1 \mathrm{eV}$ at $1 \mathrm{ps}$. The overall KER is still slightly higher compared with the two-body breakup since here Coulomb potentials are more closely approximated. The key difference being that while both of the main peaks are well separated in the two-body case, the peaks in Fig. $4 \mathrm{~b}$ are much broader, leading to an overlap of the dominant spectral features. This is due to the additional broadband energy both, the remaining $\mathrm{CH}^{+}$, and the $\mathrm{C}^{+}$fragments gather when the proton is removed from either $\mathrm{CH}_{2}^{+}$or $\mathrm{CH}^{+}$. The temporal evolution of the KER by the single fragments is shown in Supplementary Fig. 2, which indicates that apart from the $\mathrm{C}-\mathrm{C}$ bond the $\mathrm{C}-\mathrm{H}$ bond dissociates. The low-energy peak in Fig. 4 follows precisely the evolution that was observed for the peak assigned to dissociation in the two-body breakup in Fig. 3. This constitutes strong evidence that we observe the same cation dynamics in the two-body and in the three-body breakup. The KER spectra for the three-body breakup are too broad to clearly observe the highenergy shoulder associated with the launching of proton migration. However, since dissociation in the cationic states requires the absorption of a fifth photon, compared with the four photons required to initiate proton migration dynamics on the A-state, it is most likely that the latter process as well is initiated on the cation and probed via ionization to the triply charged states.

In the three-body breakup channel, we have access to the direction in which the proton is emitted, allowing us to follow the proton migration process in detail. At its simplest, if the proton trajectory is close to the direction of the $\mathrm{CH}^{+}$fragment, it means that the molecule was close to the vinylidene isomer while undergoing Coulomb explosion. While, if the proton is close to the $\mathrm{C}^{+}$fragment direction, the molecule is closer to acetylene. Such an analysis allows for the construction of a molecular movie of the proton migration reaction in momentum space. Key frames of this molecular movie are represented by the Newton plots in Fig. 5 as described in the figure caption and the Methods section. Since proton migration in the acetylene cation initiated on the A-state results in a bound vinylidene cation (that is, nondissociative), its Coulomb explosion will yield in a high KER. Therefore, the dynamics of interest were isolated by filtering the total three-body KER data, selecting only those correlated events above $13 \mathrm{eV}$, thereby excluding the $\mathrm{C}-\mathrm{C}$ bond dissociation events visible in Figs 3 and 4 . The cutoff for energy filtering is indicated as a black line in Fig. 4a. For comparison, unfiltered Newton plots are presented in Supplementary Fig. 3. In Fig. 5, proton fragment momenta are presented in the molecular frame ${ }^{33}$, in concert with classically calculated values (shown as symbols) that assume Coulomb potentials and are included to serve as a visual guide. Since the triply charged states populated in the experiment substantially deviate from a Coulomb potential, the observed momentum in the experiment is lower than the classically calculated one. Despite this deviation, the agreement between this simple calculation and the experimental data is very good. Starting from the linear geometry of neutral acetylene in the ground state, the trans-configuration is reached after $20 \mathrm{fs}$, indicated by a depletion in signal at $(x=-1.0 \mid y=0.0)$, which is shown in blue and an increased signal at $(x=-0.3 \mid y=0.7)$ shown in green. This matches a half-period of the trans-bent vibrational mode. At $40 \mathrm{fs}$, the centre of mass reaches the classically calculated transition state $(x=0.0 \mid y=1.0)$. This agrees with timescales predicted by our semi-classical simulations for a population transfer to the ground state mediated by the conical intersection. A maximum in vinylidene formation (classically calculated value $(x=0.6 \mid y=0.8))$ is observed around $100 \mathrm{fs}$, after which the population maximum swings back to the acetylene side (with a vinylidene population minimum $\sim 150 \mathrm{fs}$ ). This oscillation continues with the next vinylidene population maximum observed at $180 \mathrm{fs}$. All time values are associated with an error of $\pm 5 \mathrm{fs}$.

The to and fro isomerization behaviour of Fig. 5 is perfectly reproduced by the $a b$ initio trajectory simulations of the vibronic dynamics as evinced in Fig. 6 . While a single recurrence had also been observed in $\mathrm{C}_{2} \mathrm{D}_{2}^{2}+$ (refs 12,13), we now see further oscillations associated with the evolution of the nuclear wavepacket. We deduce from these results the following proton migration process: Both X- and A-states are populated in a threeand four-photon process, respectively; (Fig. 1). According to our calculations and ref. 22, the A-state population is depleted by roughly half in the first $40-50 \mathrm{fs}$ via a trans-bent conical 


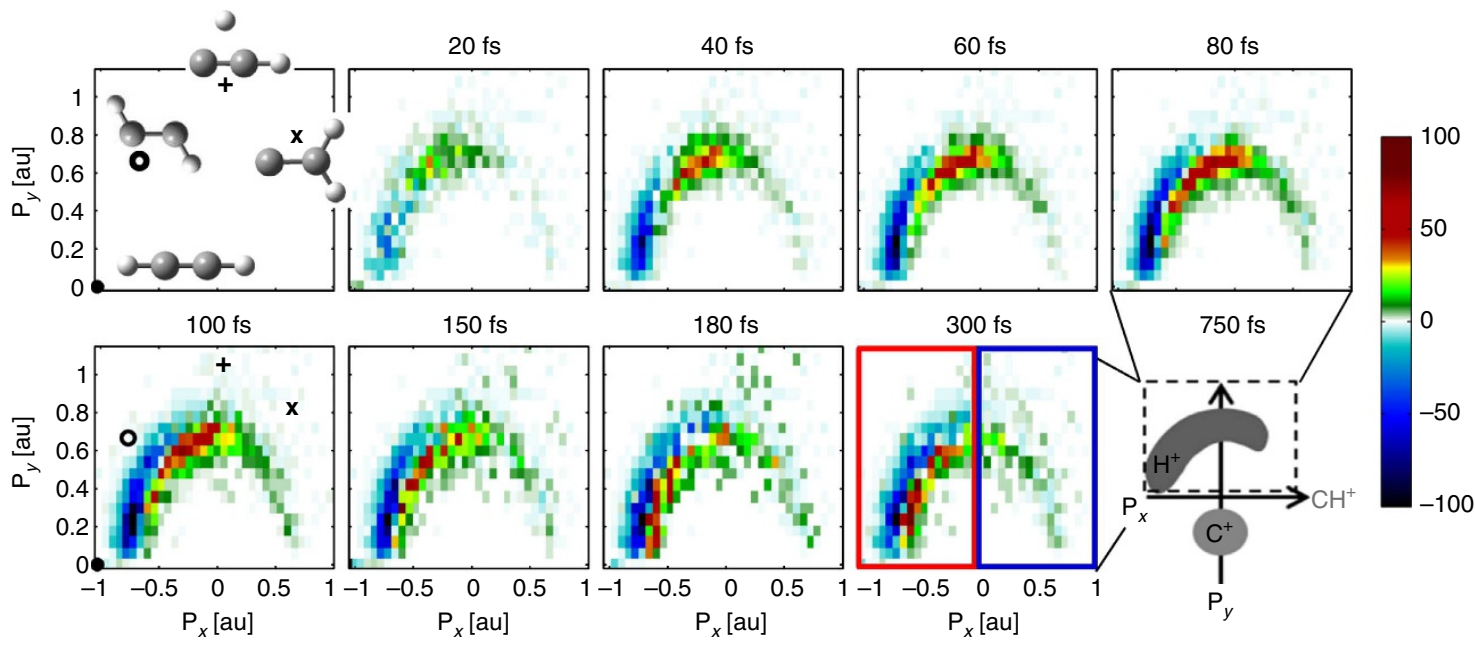

Figure 5 | The molecular movie of proton migration: Newton plots filtered for KER $>13 \mathrm{eV}$. Symbols show classically calculated results assuming Coulomb potentials for linear configuration $(\cdot)$, trans-configuration (o), transition state $(+)$ and vinylidene $(x)$, given in the upper left corner. As indicated on the lower right, all molecules are rotated such that the momentum vector of $\mathrm{CH}^{+}$points towards the positive $x$ axis, the relative momentum of $\mathrm{C}^{+}$is confined to the negative $y$ axis. Here, we plot only data of the momentum vector of $\mathrm{H}^{+}$after subtracting the distribution at $\Delta t=0$. The plots show the evolution of $\mathrm{H}^{+}$momenta with increasing time delay from 0 to $300 \mathrm{fs}$, normalized to the integral. The distribution appears localized at $20 \mathrm{fs}$ (dark green data points) and spreads out towards the $\mathrm{CH}^{+}$ion with increasing time delay. $\mathrm{C}^{+}$is considerably heavier than $\mathrm{H}^{+}$and thus localized, while the light $\mathrm{H}^{+}$ propagates with time being transferred from the acetylene configuration to the vinylidene one. White colours correspond to zero, blue colours to negative contributions (after subtraction, that is, where the population originates) and other colours to positive contributions (that is, where the population is going).

intersection to hot vibrational states of the ground electronic state of $[\mathrm{HC}=\mathrm{CH}]^{+}$. Formation of vinylidene takes place exclusively here, where the isomerization barrier can be overcome in both directions, which is why we observe the to and fro isomerization between acetylene and vinylidene (green arrow in Fig. 1). The initial X-state population does not contribute to isomerization since its energy is too low to overcome the isomerization barrier. The isomerization process itself is 'primed' by the pre-eminence of motion along the trans-bending mode in the excited state (Supplementary Figs 4-6). The solid lines in Fig. 6 represent the total ground- and excited-state populations of acetylene (red) and vinylidene (blue) cation, as elucidated in Supplementary Fig. 6. As evinced in Fig. 6, the agreement is excellent up to $150 \mathrm{fs}$. From a single exponential fit, we estimate the initial isomerization time to be $\sim 41 \mathrm{fs}$ (theory) and $43 \pm 10 \mathrm{fs}$ (experiment), which is in accord with previous results ${ }^{14}$. The temporal resolution achieved results from the fact that both the pump and the probe step are highly nonlinear (Supplementary Fig. 7).

Initial state assignment (II). In order to validate the proposed interpretation of the three-fragment correlation data, we confirm that the imaged dynamics occur on the state of interest, that is, the A-state of the cation. Evidence for a low-lying electronic state of $\Sigma$ symmetry (such as the A-state) has already been presented in the single-pulse experiments of Fig. 2 with the appearance of a high-energy shoulder analogous to observations at the FEL and its angular dependence, which reveals ionization from the HOMO-1. While Coulomb explosion occurs in the triply charged molecule $\mathrm{C}_{2} \mathrm{H}_{2}^{3+}$, there exist multiple combinations of pump- and probepulses that may be used to reach this ionic state. To verify that it is indeed dynamics on the cationic state that are being probed rather than the neutral or the subsequently prepared dicationic surface, we first note that we expect negligible dicationic populations to be prepared by the pump step since the ratio of parent ion $\mathrm{C}_{2} \mathrm{H}_{2}^{+}$to $\mathrm{C}_{2} \mathrm{H}_{2}^{2+}$ in the time of flight (TOF) spectrum is $>100: 1$, demonstrating that the pump step strongly favours

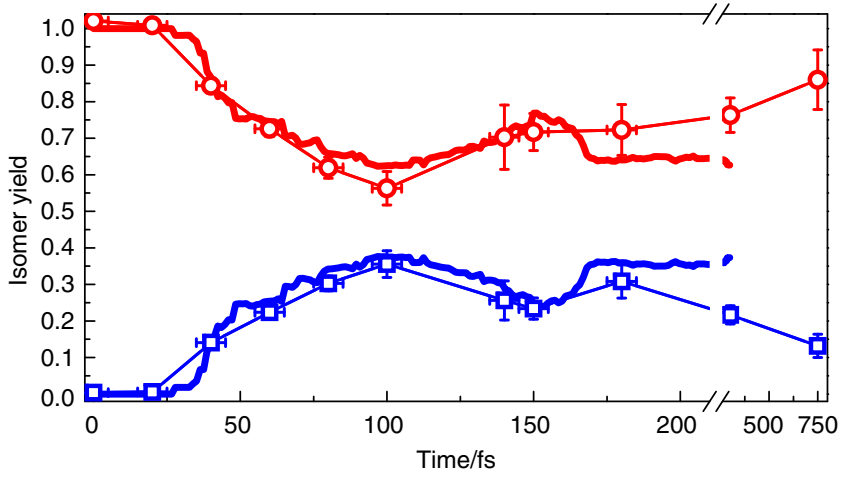

Figure 6 | Comparison of Theory and Experiment. Comparison of theory (thick lines) and experiment (open symbols) for acetylene (red) and vinylidene (blue) yield: Theoretical curves show the superposition of acetylene and vinylidene yield in both the ground state and excited states of the cation as a function of time (see Supplementary Fig. 6 for details). Experimental values are obtained by integrating the Newton plots of Fig. 5 for negative $P_{x}$ (acetylene, circles) or positive $P_{x}$ (vinylidene, squares), as indicated there by the red and blue box at $300 \mathrm{fs}$. Note the broken axis for long time delays. Experimental data are corrected by offset and scaling factor to fit the theoretical points.

preparation of the cation over the dication. In addition, neither pump- nor probe-pulses alone contribute $>0.3 \%$ to the correlated counts of the two-body breakup compared with their joined interaction. Of course, our method is only sensitive to the combination of pump and probe step, and thus an extremely efficient (but here undesirable) probe step for the transition from the $2^{+} \rightarrow 3^{+}$states compared with the $1^{+} \rightarrow 2^{+}$could, in principle, compensate for the pump efficiency. We therefore estimated the tunnel ionization rates for these processes based on the Keldysh theory (Supplementary Discussion). The results show the $1^{+} \rightarrow$ $2^{+}$ionization process to be clearly saturated, which holds for the intensity ranges accessible during our experiment. This means 
that the preference of populating the cation over the dication in the pump step is preserved in the probe step such that the overwhelming cation population created by the pump is the one imaged by the probe step. This is consistent with (and perhaps a necessary precondition for) the observation of the triply charged species. Combining the efficiencies of pump and probe step, it appears therefore extremely unlikely that we observe dication dynamics in the case of three-fragment correlation, which is also supported by the fact that the same dissociation dynamics is observed both in the dication (Fig. 3) and the trication (Fig. 4), as discussed earlier. Further, dynamics in neutral $\mathrm{C}_{2} \mathrm{H}_{2}$ can also be excluded on the basis of the power dependence measurement of the single 266-nm pulse experiment presented in Supplementary Fig. 8. While for low pump powers, we observe a four-photon absorption that matches the ionization potential of the A-state energetically, for higher pump powers it is indicated that we are driving the pump step in saturation. This saturation effect also holds for the experimental condition of the pump-probe experiment. Thus, by the arrival time of the probe-pulse, there are no neutral molecules left in the jet. In addition, the identity of the KER of a $\mathrm{CH}^{+}$fragment from the two-body breakup with an uncorrelated $\mathrm{CH}^{+}$fragment, as discussed in Supplementary Fig. 9, further indicates that we are probing dynamics in the cation. Finally, this assignment is strongly supported by the excellent agreement with new high-level theoretical simulations of the $\mathrm{C}_{2} \mathrm{H}_{2}^{+}$A-state initiated dynamics, as shown in Fig. 6 .

In summary, if one aims to populate the excited states of charged molecules whose difference in ionization potentials is too large to be overcome by $800 \mathrm{~nm}$ photons, our experimentally rather simple approach provides an alternative to demanding VUV sources. Indeed, given the limited availability, repetition rate, pulse stability and timing jitter of FELs, our approach provides tremendous benefits in terms of statistics and temporal resolution. Combining pump-probe CEI with multiphoton absorption of UV light opens the door to time-resolved imaging of chemical reactions and very rich dynamics since a variety of electronic states is accessed simultaneously. Careful consideration, as here, makes it possible to identify their origins unambiguously. Combined with theoretical simulations, our results present the most complete picture of proton migration and $\mathrm{C}=\mathrm{C}$ bond breaking on the ground and excited states of $\mathrm{C}_{2} \mathrm{H}_{2}^{+}$to date.

\section{Methods}

Experimental technique. Experiments were carried out at the Advanced Laser Light Source ALLS (INRS-EMT, Varennes, Canada). We use pump-probe CEI: a pump-pulse $(266 \mathrm{~nm}, 32 \mathrm{fs}$ and $3.2 \mu \mathrm{J})$ ionizes the system to the cation and launches proton migration dynamics. A time-delayed probe-pulse $(800 \mathrm{~nm}, 40 \mathrm{fs}$ and $44 \mu \mathrm{J})$ further ionizes it to higher charged states. Its electric field stripes off electrons almost immediately, leaving positively charged fragments behind that undergo Coulomb explosion. They represent the molecule's geometric configuration at the arrival time of the probe-pulse. The strength of CEI is its ability to directly image geometrical structures. It does not provide direct observation of electronic states, as photoelectron spectroscopy would do. Nevertheless, we are able to draw conclusions about the electronic states populated in the initial ionization from the angular distribution of the molecular fragments and theoretical support.

Intense $266 \mathrm{~nm}$ pulses were obtained by sum frequency generation of the fundamental beam $(800 \mathrm{~nm}, 2.5 \mathrm{KHz}$ and $35 \mathrm{fs}$, KM labs) with its second harmonic $(400 \mathrm{~nm})$ in a $40-\mu \mathrm{m}$ thick-type I BBO crystal (Altos Photonics). Pulse duration was controlled by chirped mirrors (Ultrafast Innovation) and/or fused silica plates from almost Fourier-transform limited ( $32 \mathrm{fs}$ ) to above $250 \mathrm{fs}$ by adding a positive chirp. Measurements were obtained with a homebuilt TG-FROG ${ }^{34}$. Pulse energy was varied between 11.2 and $3.2 \mu \mathrm{J}$. For the pump-probe experiments, a $32-\mathrm{fs}$, $3.2 \mu \mathrm{J}$ pump-pulse of $266 \mathrm{~nm}$ was combined with a $40-\mathrm{fs}, 44 \mu \mathrm{J}$ probe-pulse of $800 \mathrm{~nm}$. Laser polarization was vertical for both, pump- and probe-beams. Laser pulses were focussed $(f=100 \mathrm{~mm})$ into a collimated acetylene gas jet (Praxair AC 2.6AA-A5). Pump intensity on target was estimated to $3 \times 10^{14} \mathrm{~W} \mathrm{~cm}^{-2}$ for the pump-probe experiment. Ionic fragments resulting from Coulomb explosion are collected with a uniform-electric-field ion imaging spectrometer. Their full threedimensional momenta are retrieved using a time- and position-sensitive delay line detector at the end of the $23-\mathrm{cm}$ ion TOF spectrometer (RoentDek Handels $\mathrm{GmbH})$. Owing to the vertical polarization direction two fragments of same mass and charge but opposite momenta (for example, $\mathrm{CH}^{+} / \mathrm{CH}^{+}$, the acetylene channel) will hit the same detector position, but at different times. Thus choosing the correct TOF range enables us to distinguish them.

Owing to a significantly increased intensity when pump- and probe-pulses overlap temporally, the effective focal volume is increased within which the threshold for ionization is reached, leading to an increase in ionized molecules. This allows determining time zero by the appearance of a $60 \%$ increase in total ion yield (sum of all ion fragments) when scanning the delay stage. Error of time zero assignment is around $\pm 5 \mathrm{fs}$, partially due to drifts over typical acquisition times of 24-48 h. Even though the pulse durations of 32 and $40 \mathrm{fs}$ for pump- and probepulses appear quite long, we like to point out that both-pump and probe step-are highly nonlinear. A four-photon excitation effectively shortens the pump-pulse to $16 \mathrm{fs}$, and also the effective tunnel ionization time for the probe-pulse will be much shorter than the actual pulse duration.

Data analysis. Owing to the large count rates produced in this experiment (typically $>3$ ions per laser shot), a sophisticated algorithm is used to parse the data for true coincidences ${ }^{35}$. A TOF window is defined in order to identify events registered by the position-sensitive detector as specific ions-either $\mathrm{H}^{+}, \mathrm{C}^{+}$, $\mathrm{CH}^{+}, \mathrm{CH}_{2}^{+}$or $\mathrm{CC}^{+}$. The data is then parsed, laser shot by laser shot, for the existence of a specific fragmentation channel, for example, $\left(\mathrm{H}^{+}, \mathrm{C}^{+}\right.$and $\left.\mathrm{CH}^{+}\right)$ for the three- or for the two-body breakup $\left(\mathrm{CH}^{+}\right.$and $\left.\mathrm{CH}^{+}\right)$, as the acetylene channel and $\left(\mathrm{C}^{+}\right.$and $\left.\mathrm{CH}_{2}^{+}\right)$giving the vinylidene channel. If data from a laser shot yield the desired ions for the fragmentation channel, the momentum for these ions is calculated. If the momentum sum is near zero $\left(<10^{-23} \mathrm{~kg} \mathrm{~m} \mathrm{~s}^{-1}\right)$, the group of ions is considered as a true coincidence, originating from the same molecule. The momentum information for this channel is stored for further calculation of various metrics, for example, KER, $\theta$ (inset of Fig. 1) and so on. It is possible with these high count rates that multiple ions are detected in the TOF windows defined for a specific channel, leading to several possible combinations of ions-the majority of which are false. In this case, all ion combinations are considered and only that which yields near zero momentum is stored as a true coincidence. Each laser shot excites a neutral molecule in the ultrasonic jet. From this molecule, we detect the full-fragment momentum information $\left(\mathrm{CH}^{+}, \mathrm{C}^{+}\right.$and $\mathrm{H}^{+}$) in coincidence. This experiment is repeated many thousand times. All this information together is fed into the Newton plots that gives us the molecular movie. This represents not only a standard experimental approach but also a prerequisite to detect the square of a quantum mechanical wavepacket. So every point in the Newton plot originates from a single detected molecule where all molecules started with the same initial conditions.

Theoretical methods. The excited non-adiabatic molecular dynamics on the $A^{2} \Sigma_{g}^{+}$state were simulated using the full-multiple spawning approach ${ }^{36}$, a semiclassical method. In this methodology, the vibronic wavepacket is represented in a basis of direct products (over each Cartesian coordinate) of frozen Gaussian functions. These basis functions, or trajectories, are propagated classically, with the potential energy surfaces determined 'on-the-fly' using $a b$ initio electronic structure methods. The number of trajectories expands dynamically, to account for regions of non-adiabatic coupling between electronic states, by 'spawning' (approximating nonadiabatic transitions) new basis functions onto different electronic states as needed. The requisite energy gradients and derivative couplings required to propagate the trajectories were computed at a very high level of electronic structure theory, using atomic natural orbital basis sets and a first-order multireference configuration interaction treatment of electron correlation ${ }^{37}$. The underlying complete active space reference functions for the multireference configuration interaction procedure were determined using a 7 electron, 7 orbital $(7 e, 7 o)$ active space. The initial distribution of nuclear positions and momenta for the simulation were generated by sampling the ground vibrational state of neutral acetylene ${ }^{38}$. From 20 initial conditions, 1,480 spawned trajectory basis functions were generated. On the basis of these simulations, the adiabatic state populations as a function of time, as well as the geometric character of the wavepacket (that is, acetylenic versus vinylidenic) could be readily determined. Details are provided in the Supplementary Methods.

Assuming a Coulomb potential for the $3+$ charge state $\left(\mathrm{C}_{2} \mathrm{H}_{2}^{3+} \rightarrow\right.$ $\mathrm{C}^{+}+\mathrm{CH}^{+}+\mathrm{H}^{+}$), momentum positions within the Newton plot have been calculated for the following four structures using classical mechanics: (1) $\mathrm{C}_{2} \mathrm{H}_{2}$ at equilibrium; (2) $\mathrm{C}_{2} \mathrm{H}_{2}^{+}$in the trans-geometry; (3) $\mathrm{C}_{2} \mathrm{H}_{2}^{+}$at the transition state at the conical intersection; and (4) $\mathrm{C}_{2} \mathrm{H}_{2}^{+}$in vinylidene geometry. For the $\mathrm{CH}^{+}$ fragment, it is assumed that the charge is located on the carbon with a mass of 13. The initial velocities of the fragments are assumed to be zero. Geometrical structures were obtained using the level of theory described above.

The angular dependence of the ionization rate from HOMO and HOMO-1 was calculated using TDDFT ${ }^{39}$. Bond length have been set to their optimized neutral parameters value, that is, $\mathrm{HCCH}$ geometries parameters are $\mathrm{CH}=0.110 \mathrm{~nm}$ and $\mathrm{CC}=0.122 \mathrm{~nm}$. The $\mathrm{C}-\mathrm{C}$ bond was set to be parallel to the $z$ axis. For each spin orbital, Kohn-Sham molecular orbital, the time-dependent ionization probability was calculated. From this, we have extracted the ratio of HOMO-1 and HOMO presented in Fig. 2b). Further details are provided in the Supplementary Methods. 


\section{References}

1. Zewail, A. H. Four-dimensional electron microscopy. Science 328, 187-193 (2010).

2. Weinstein, J. A. \& Hunt, N. T. In search of molecular movies. Nat. Chem. 4, 4-5 (2012).

3. Blaga, C. I. et al. Imaging ultrafast molecular dynamics with laser-induced electron diffraction. Nature 483, 194-197 (2012).

4. Haessler, S. et al. Attosecond imaging of molecular electronic wavepackets. Nat. Phys. 6, 200-206 (2010).

5. Wörner, H. J., Bertrand, J. B., Kartashov, D. V., Corkum, P. B. \& Villeneuve, D. M. Following a chemical reaction using high-harmonic interferometry. Nature 466, 604-607 (2010).

6. Chapman, H. N. et al. Femtosecond X-ray protein nanocrystallography. Nature 470, 73-77 (2011)

7. Miller, R. J. D. et al. 'Making the molecular movie': first frames. Acta Crystallogr. A 66, 137-156 (2010).

8. Eichberger, M. et al. Snapshots of cooperative atomic motions in the optical suppression of charge density waves. Nature 468, 799-802 (2010).

9. Barty, A., Küpper, J. \& Chapman, H. N. Molecular imaging using X-ray freeelectron lasers. Annu. Rev. Phys. Chem. 64, 415-435 (2013).

10. Légaré, F. et al. Imaging the time-dependent structure of a molecule as it undergoes dynamics. Phys. Rev. A 72, 1-4 (2005).

11. Bocharova, I. et al. Charge resonance enhanced ionization of $\mathrm{CO}_{-}\{2\}$ probed by laser coulomb explosion imaging. Phys. Rev. Lett. 107, 63201 (2011).

12. Matsuda, A., Fushitani, M., Takahashi, E. J. \& Hishikawa, A. Visualizing hydrogen atoms migrating in acetylene dication by time-resolved three-body and four-body Coulomb explosion imaging. Phys. Chem. Chem. Phys. 13, 8697-8704 (2011).

13. Hishikawa, A., Matsuda, A., Fushitani, M. \& Takahashi, E. J. Visualizing recurrently migrating hydrogen in acetylene dication by intense ultrashort laser pulses. Phys. Rev. Lett. 99, 258302 (2007).

14. Jiang, Y. H. et al. Ultrafast extreme ultraviolet induced isomerization of acetylene cations. Phys. Rev. Lett. 105, 263002 (2010).

15. Koopmans, T. Über die Zuordnung von Wellenfunktionen und Eigenwerten zu den Einzelnen Elektronen Eines Atoms. Physica 1, 104-113 (1934).

16. McFarland, B. K., Farrell, J. P., Bucksbaum, P. H. \& Gühr, M. High harmonic generation from multiple orbitals in N2. Science 322, 1232-1235 (2008).

17. Akagi, H. et al. Laser tunnel ionization from multiple orbitals in $\mathrm{HCl}$. Science 325, 1364-1367 (2009).

18. Smirnova, O. et al. High harmonic interferometry of multi-electron dynamics in molecules. Nature 460, 972-977 (2009).

19. Tilborg, J. Van. et al. Femtosecond isomerization dynamics in the ethylene cation measured in an EUV-pump NIR-probe configuration. J. Phys. B At. Mol. Opt. Phys. 42, 081002 (2009).

20. Takahashi, E. J., Lan, P., Mücke, O. D., Nabekawa, Y. \& Midorikawa, K. Attosecond nonlinear optics using gigawatt-scale isolated attosecond pulses. Nat. Commun. 4, 2691 (2013).

21. Tzallas, P., Skantzakis, E., Nikolopoulos, L. a. a., Tsakiris, G. D. \& Charalambidis, D. Extreme-ultraviolet pump-probe studies of onefemtosecond-scale electron dynamics. Nat. Phys. 7, 781-784 (2011).

22. Madjet, M. E., Li, Z. \& Vendrell, O. Ultrafast hydrogen migration in acetylene cation driven by non-adiabatic effects. J. Chem. Phys. 138, 094311 (2013).

23. Douguet, N., Rescigno, T. N. \& Orel, a. E. Time-resolved molecular-frame photoelectron angular distributions: snapshots of acetylene-vinylidene cationic isomerization. Phys. Rev. A 86, 013425 (2012).

24. Jiang, Y. H. et al. Ultrafast dynamics in acetylene clocked in a femtosecond XUV stopwatch. J. Phys. B At. Mol. Opt. Phys 46, 164027 (2013).

25. Wells, E. et al. Adaptive strong-field control of chemical dynamics guided by three-dimensional momentum imaging. Nat. Commun. 4, 2895 (2013).

26. Alnaser, A. et al. Momentum-imaging investigations of the dissociation of D $2+$ and the isomerization of acetylene to vinylidene by intense short laser pulses. J. Phys. B At. Mol. Opt. Phys. 39, S485-S492 (2006).
27. Boyé-Péronne, S., Gauyacq, D. \& Liévin, J. Vinylidene-acetylene cation isomerization investigated by large scale ab initio calculations. J. Chem. Phys. 124, 214305 (2006).

28. Lezius, M. et al. Nonadiabatic multielectron dynamics in strong field molecular ionization. Phys. Rev. Lett. 86, 51-54 (2001).

29. Madjet, M. E.-A., Vendrell, O. \& Santra, R. Ultrafast dynamics of photoionized acetylene. Phys. Rev. Lett. 107, 263002 (2011).

30. Telnov, D. a. \& Chu, S.-I. Effects of electron structure and multielectron dynamical response on strong-field multiphoton ionization of diatomic molecules with arbitrary orientation: An all-electron time-dependent densityfunctional-theory approach. Phys. Rev. A 79, 041401 (2009).

31. Zyubina, T. S., Dyakov, Y. a., Lin, S. H., Bandrauk, a. D. \& Mebel, a. M. Theoretical study of isomerization and dissociation of acetylene dication in the ground and excited electronic states. J. Chem. Phys. 123, 134320 (2005).

32. Xu, H. et al. Two-proton migration in 1,3-butadiene in intense laser fields. Phys. Chem. Chem. Phys. 12, 12939-12942 (2010).

33. Wales, B. et al. Multiple ionization and complete fragmentation of OCS by impact with highly charged ions Ar $4+$ and Ar $8+$ at $15 \mathrm{keV} \mathrm{q} \mathrm{-} \mathrm{1.} \mathrm{J.} \mathrm{Phys.} \mathrm{B}$ At. Mol. Opt. Phys. 45, 045205 (2012).

34. Schmidt, B. E. et al. Poor man's source for sub 7 fs: a simple route to ultrashort laser pulses and their full characterization. Opt. Express 16, 18910-18921 (2008).

35. Wales, B. et al. A coincidence detection algorithm for improving detection rates in Coulomb Explosion Imaging. Nucl. Instrum. Methods Phys. Res. Sect. A Accel. Spectrometers Detect. Assoc. Equip 667, 11-15 (2011).

36. Ben-Nun, M. \& Martínez, T. J. Ab initio quantum molecular dynamics. $A d v$. Chem. Phys. 121, 439-512 (2002).

37. Lischka, H. et al.COLUMBUS, an ab initio electronic structure program, release 7.0 (2012); Available at http://www.univie.ac.at/columbus/.

38. Stanton, J. F. et al. CFOUR, a quantum chemical program package. Available at http://www.cfour.de.

39. Ullrich, C. A. \& Bandrauk, A. D. in Time-Dependent Density Funct. Theory SE - 24 Vol. 706 (eds Marques, M. L. et al.) 357-375 (Springer Berlin Heidelberg, 2006).

\section{Acknowledgements}

We gratefully acknowledge research funding from NSERC (Natural Sciences and Engineering Research Council of Canada), FQRNT (Fonds de recherche du Québec Nature et technologies) and CIPI (Canadian Institute for Photonic Innovations). H.I acknowledges financial support from the NSERC-Banting Postdoctoral Fellowships Program. S.B. and V.W. are thankful for financial support from NSERC.

\section{Author contributions}

H.I. and F.L. designed the experiment; H.I., B.W., S.B., B.E.S., N.T., E.B., M.G., C.T.H and J.-C.K. carried out the experiments; M.S.S., E.P.F., A.D.B., M.S. and F.L. provided the theoretical support; H.I., B.W., S.B., V.W., M.S.S., J.S. and F.L. carried out data analysis and interpretation; H.I., F.L., M.S.S. and J.S. wrote the manuscript with support from all other authors.

\section{Additional information}

Supplementary Information accompanies this paper at http://www.nature.com/ naturecommunications

Competing financial interests: The authors declare no competing financial interests.

Reprints and permission information is available online at http://npg.nature.com/reprintsand permissions.

How to cite this article: Ibrahim, H. et al. Tabletop imaging of structural evolutions in chemical reactions demonstrated for the acetylene cation. Nat. Commun. 5:4422 doi: 10.1038/ncomms5422 (2014). 